\title{
Design and Characterization of Superlattice Infrared Photodetector Operating at Low Bias Voltage
}

\author{
Mao-Chieh Hsu, Student Member, IEEE, Yao-Fong Hsu, Shih-Yan Lin, and Chieh-Hsiung Kuan, Member, IEEE
}

\begin{abstract}
In this paper, we investigate the performance and characterization of a 15-period superlattice embedded between two thick AlGaAs barriers. The structure can operate at low bias voltage with less power consumption for 8-10 $\mu \mathrm{m}$ long-wavelength infrared detection. In our design, one barrier is used to reduce the dark current and the other one is designed to enhance the collection efficiency of photoelectrons at the collector contact. The fabricated detector can be operated at a bias voltage lower than $0.1 \mathrm{~V}$ and exhibits pronounced photovoltaic response. The spectral response shows voltage dependence around $0 \mathrm{~V}$. At high bias voltage $(>25 \mathrm{mV})$ the spectral lineshape is independent of bias and is around 8-10 $\mu \mathrm{m}$ with peak wavelength at $9.3 \mu \mathrm{m}$. At lower bias voltage the response is shifted toward shorter wavelength range. The peak responsivity was found to be $12 \mathrm{~mA} / \mathrm{W}$ at $\lambda_{p}=8.7 \mu \mathrm{m}$ and zero bias and $85 \mathrm{~mA} / \mathrm{W}$ at $\lambda_{p}=9.3 \mu \mathrm{m}$ and $0.1 \mathrm{~V}$. Background limited can be achieved up to $65 \mathrm{~K}$ with bias voltage less than $0.1 \mathrm{~V}$. The measured noise power spectral density of the dark current at $77 \mathrm{~K}$ shows the characteristics of full shot noise rather than the generation-recombination noise. The peak detectivity is determined to be $D^{*}=3.5 \times 10^{9} \mathrm{~cm} \sqrt{\mathrm{Hz}} / \mathrm{W}$ at 77 $K$ and $0.1 \mathrm{~V}$. In comparison with a conventional 30-period QWIP, our detector has the advantages of better performance at low bias voltages with lower power consumption and a tunable feature of spectral range.
\end{abstract}

Index Terms-Noise, noise measurement, photodetector, quantum-well infrared photodetector.

\section{INTRODUCTION}

$\mathbf{L}$ ONG wavelength infrared detection using intersubband transitions in quantum well structures has been under intensive investigation [1]-[6], since the first observation of intersubband transition of GaAs/AlGaAs quantum wells [7]. These intersubband infrared photodetectors can be implemented in wide bandgap materials as a result of the detectable photonenergy determined by the subband energies of the quantum well or superlattice structures rather than by the fundamental bandgap of the building materials. Due to the mature growth and processing technology of building materials, quantum well intersubband photodetectors (QWIP's) are potentially suitable for large area focal plane array (FPA). Recently, large area focal plane array (FPA) with QWIP's has been demonstrated with high uniformity and performance [8].

Using photoexcitation between localized states and the continuum, QWIP is usually operated as a photoconductive device. An external bias voltage is required to accelerate the excited

Manuscript received May 25, 1999; revised October 27, 1999. This work was supported by National Council of Republic of China under Contract NSC882112-M-002-030. The review of this paper was arranged by Editor P. K. Bhattacharya.

The authors are with the Department of Electrical Engineering, National

Taiwan University, Taipei 10617, Taiwan, R.O.C.

Publisher Item Identifier S 0018-9383(00)03401-8. carriers and generate the measurable photocurrent. Typically a QWIP consists of 30 to 50 quantum wells. Although the responsivity is insensitive to well number [9], a large well number is favorable to reduce the noise level. In addition, to reduce the tunneling and thermally assisted tunneling current [10], [11], a QWIP is typically implemented with thick barriers (300-500 $\AA$ ) [1]. As a result, a typical QWIP requires a relatively high bias voltage (1-4 V) [1], [8] to optimize its performance. For certain applications, a lower bias voltage with less power dissipation would be advantageous. Due to the formation of miniband to facilitate the transportation of excited electrons, superlattice infrared detectors (SLIP's) are expected to operate at very low bias voltage. Several designs for SLIP's have been investigated [12]-[16]. To reduce the large ground-state tunneling current, most SLIP's were designed with a thick barrier at the collector side as a current blocking layer [12]-[15]. The blocking layer is designed to allow the photoelectrons to pass but block the tunneling current. The detection mechanism of a SLIIP with such a blocking barrier is similar to that of an internal emission detector [17]. As those internal photoemission detectors, some of the reported SLIP's, with the built-in field in the blocking layer, were demonstrated as photovoltaic detectors [13], [14].

Although the reported detectivity of SLIP's seems to be inferior to that of QWIP's using thick barriers [1], we should note that the comparisons were made between the optimized QWIP's and the SLIP's without further optimization. Therefore, the performance of SLIP's might be underestimated. In this article, we investigate a modified design for superlattice infrared photodetectors. As illustrated in Fig. 1, a superlattice (SL) layer is embedded between two thick barriers. The thick barrier on the right-hand side (collector side) is a current blocking barrier. Another thick barrier with an energy height higher than the top of the excited miniband is designed to enhance the probability of photoelectron being emitted toward the collector contact. Without such a barrier, part of photoelectrons would escape from the left-hand side boundary of the SL region because photoelectrons can be emitted toward either right-hand or left-hand directions. The escaping photoelectrons would result in a lower net quantum efficiency. With this barrier, photoelectrons moving toward this barrier can be blocked and redirected into right-hand direction. In addition, the confinement of ground state carriers caused by the two thick barriers might also improve the absorption strength. Due to scattering and relaxation processes, the probability of these reflected carriers to be collected at the collector will be decreased with their travel distant. To reduce the distance, we used a 15-period SL instead of a typical 50-period one. The emitter was fabricated on the end periods of SL layer to replenish the photo-depleted SL. 


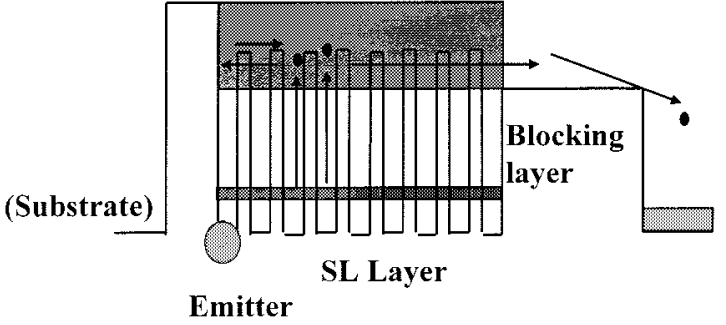

Fig. 1. Schematic energy band structure diagram for the SLIP.

The characterization of a detector based on this design will be presented. The performance of this detector will be compared to that of a 30-period QWIP which covers a similar spectral range.

\section{SAMPLE Structure, Growth AND Processing}

The sample was grown by molecular beam epitaxy on a semiinsulating GaAs substrate. First, a $300 \AA \mathrm{Al}_{0.3} \mathrm{Ga}_{0.7}$ As barrier was grown followed by a SL layer. The SL layer consists of 15 periods of $60 \AA$ GaAs doped with $n=5 \times 10^{17} \mathrm{~cm}^{-3}$ and $30 \AA$ undoped $\mathrm{Al}_{0.3} \mathrm{Ga}_{0.7}$ As. After the SL layer is a 1500 $\AA$ undoped $\mathrm{Al}_{0.22} \mathrm{Ga}_{0.78}$ As layer. Finally, a $3000 \AA \mathrm{n}^{+}$layer $\left(n=1.2 \times 10^{18} \mathrm{~cm}^{-3}\right.$ ) is grown on top for the ohmic contact. Based on the Kronig-Penny model taking into account the position dependent effective mass, we estimate the first miniband to be located at $45-69 \mathrm{meV}$ and the second miniband to be located at $182-260 \mathrm{meV}$. The height of the blocking layer is just below the second miniband. The sample was processed into $180 \mu \mathrm{m} \times 180 \mu \mathrm{m}$ mesas by standard lithography and wet chemical etching. AuGeNi/Au were evaporated on top and bottom to form the ohmic contacts. Selective area of the sample was polished into a $45^{\circ}$ facet for a light coupling.

\section{DARK CuRrent AND BACKGRound PhotocurRent}

The current-voltage $(I-V)$ characteristics were measured by using a Keithley 236 source measurement unit. The sample was mounted on the cold head of a closed-cycle cryostat to control sample temperature. A Si-diode temperature sensor was placed beside the samples to ensure the accuracy of sample temperature. Fig. 2 shows the resulting $I-V$ curves. The solid lines were taken with a cold shield (about $77 \mathrm{~K}$ ) and within a temperature range from 35 to $85 \mathrm{~K}$ while the dashed line was at $35 \mathrm{~K}$ but with the room temperature background through a KRS-5 window. Thus, the difference between the $35 \mathrm{~K}$ solid line and dash line represents the background photocurrent. Even at zero bias voltage, we can observe a photocurrent resulting from the background radiation. For $T>65 \mathrm{~K}$, the dark current begins to be dominant over the background photocurrent. Thus, under the field of view determined by the window (roughly $2 \pi$ solid-angle field of view), the device is in background limited performance (BLIP) up to $65 \mathrm{~K}$ for bias voltage smaller than $0.1 \mathrm{~V}$.

The increase of dark current at high temperatures is primarily due to the thermal current which consists of thermionic emission and thermally assisted tunneling currents. To demonstrate this, we plot the dark current as a function of inverse temperature

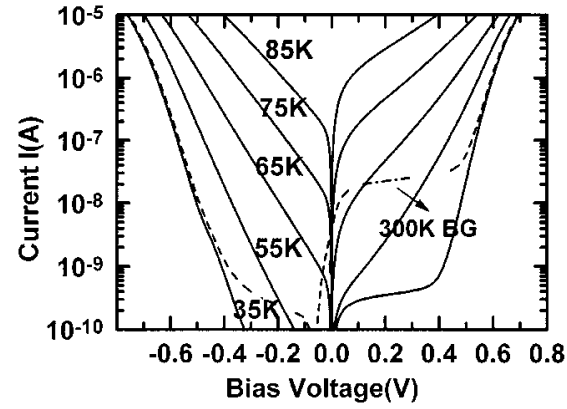

Fig. 2. Dark $I-V$ curves for the SLIP from $35 \mathrm{~K}-85 \mathrm{~K}$. The dash line is the $I-V$ curve measured at $35 \mathrm{~K}$ under room temperature background radiation. Positive voltage means top contact positive.

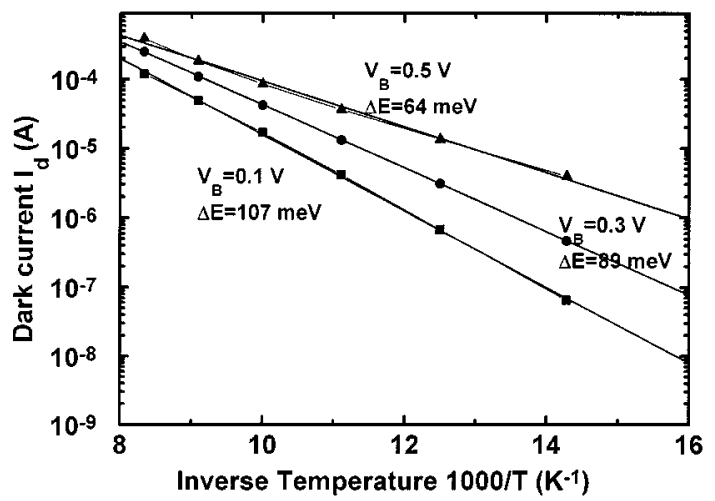

Fig. 3. Plot of dark current versus inverse temperature for three different operating bias points. The activation energy decreases with the increase of bias voltage.

at three different bias voltages in a semi-log scale as shown in Fig. 3. The linearity of the dark current in the figure indicates

$$
I_{d} \propto e^{-\Delta E / k T}
$$

where $I_{d}$ is the dark current and $\Delta E$ is the thermal activation energy. This result implies that the thermal current is dominant for $T>65 \mathrm{~K}$. In particular, the activation energy is about 107 $\mathrm{meV}$ at low bias voltage $(<0.1 \mathrm{~V})$, which is consistent with the energy difference of the blocking barrier height and the Fermi level. The dominant mechanism at the low bias range is then due to thermionic emission. With the increment of bias voltage, because of the thermally assisted mechanism, the effective height and width of the blocking barrier decrease and the resulting activation energy decreases dramatically.

\section{RESPONSIVITY MEASUREMENTS}

The spectral response was measured using Perkin Elmer 2000 Fourier transform infrared (FTIR) spectrometer. The absolute value of the responsivity was determined by measuring the blackbody responsivity with a calibrated blackbody source. Fig. 4 shows the spectral responsivity measured at $77 \mathrm{~K}$ under several different biases. For the bias voltage $>25 \mathrm{mV}$, the response spectrum has one pronounced peak around $9.3 \mu \mathrm{m}$ and its lineshape is insensitive to bias voltage. The lineshape shows a shoulder-like feature at short wavelength range. Similar lineshapes have also been observed in the reported absorption spectra of other superlattice structures [18]. The 


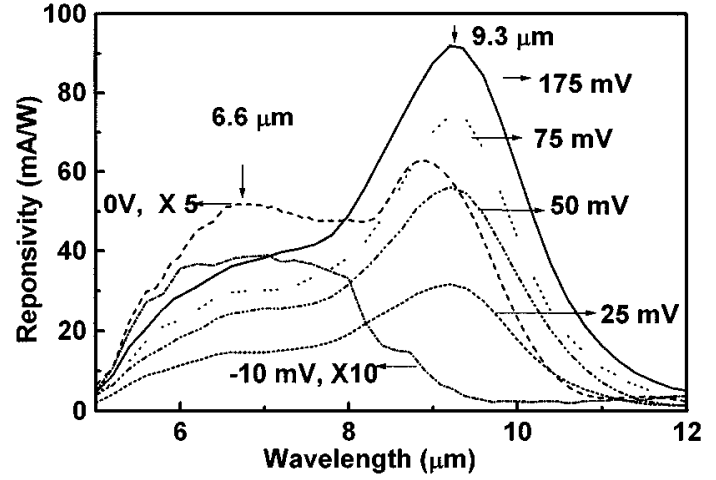

Fig. 4. Spectral response measured at several bias voltages. The lineshape at high biases $(>25 \mathrm{mV})$ is insensitive to bias voltage, while around $0 \mathrm{~V}$ the peak wavelength is shifted toward high-energy position.

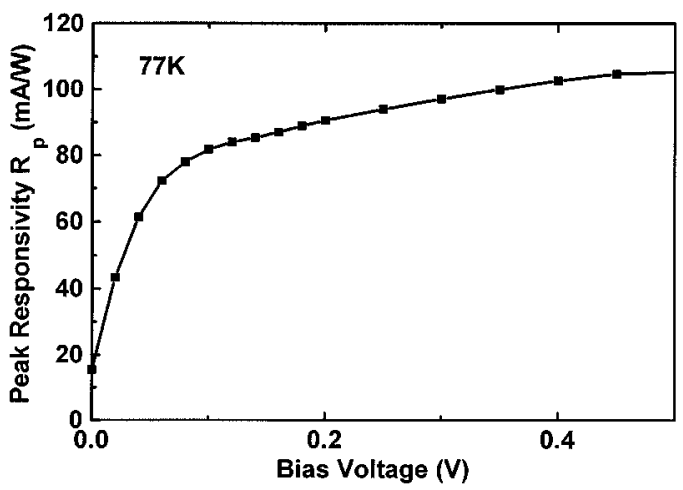

Fig. 5. Voltage dependence of peak responsivity at $77 \mathrm{~K}$.

shoulder is attributed to the transitions near the miniband zone center (i.e., the $\Gamma$ point). It is noted the lineshape of response spectra has a dramatic change at around zero bias when the bias voltage of the detector is changed from the positive bias toward the negative bias. The voltage dependence of spectral response near zero bias voltage can be explained by the effect of the blocking layer. Because the Fermi level in SL layer is higher than that of collector contact layer, we expect the energy height of the blocking layer is tilted up on the collector side at zero bias voltage. As a result, the energy height of the blocking layer is above the bottom of second miniband and those photoelectrons excited into the miniband edge are blocked by the tilted blocking layer near zero bias voltage. The effect becomes more obvious when the detector is under slightly negative bias voltage. As we can observe from the curve of -10 $\mathrm{mV}$ in Fig. 4, the low energy peak disappears and only those high-energy photoelectrons contribute to the photoresponse. Fig. 5 shows the voltage dependence of peak responsivity. The peak responsivity is $12 \mathrm{~mA} / \mathrm{W}$ at $0 \mathrm{~V}$ and is $85 \mathrm{~mA} / \mathrm{W}$ at 0.1 $\mathrm{V}$. After $0.1 \mathrm{~V}$ the increasing rate becomes sluggish, and the responsivity is saturated at a value about $105 \mathrm{~mA} / \mathrm{W}$ after 0.45 $\mathrm{V}$. The responsivity under reverse bias voltages is almost 20 times smaller than that under forward bias voltages, as can be seen by the background photocurrent in Fig. 2 .

\section{NOISE PERFORMANCE}

The noise current power spectral density was measured through a low noise current amplifier (SR570) connected to an

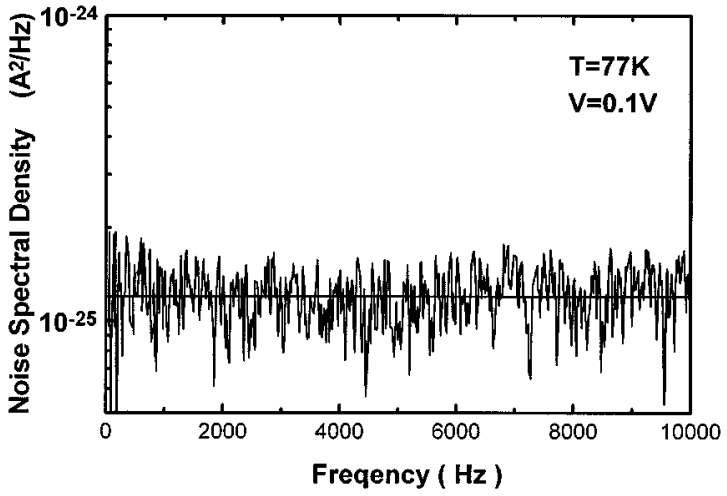

Fig. 6. Noise spectral density versus frequency. The spectrum is flat and an average value is taken as the associated PSD.

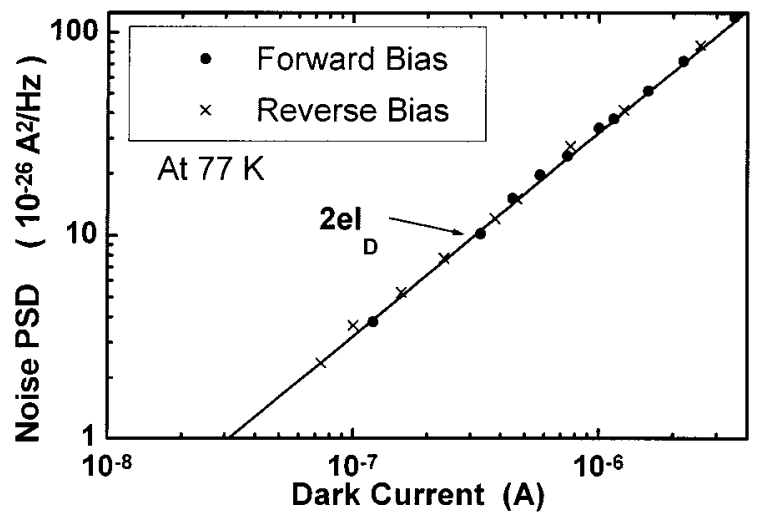

Fig. 7. Noise power spectral density versus dark current at $77 \mathrm{~K}$. The Johnson noise has been subtracted from the noise power spectrum.

FFT spectrum analyzer (AD9112B), and the bias was supplied by a $\mathrm{Nd} / \mathrm{Cd}$ battery with resistor network. After deducting the noise contribution from the amplifier and bias resistors the noise current power spectral density (PSD) of a device can be obtained. The analyzed frequency range is $0-10 \mathrm{KHz}$, which is limited by the bandwidth of the low noise amplifier. A typical measurement of the noise current PSD of the device is shown in Fig. 6. It shows a plateau of PSD in our measured frequency range.

After subtracting the Johnson noise from noise current PSD, the PSD versus dark current is plotted in Fig. 7. The result suggests that the noise current power spectral density $S_{i}$ of the dark current is

$$
S_{i}=\frac{i_{n}^{2}}{B}=2 e I_{d}
$$

where $i_{n}$ is the noise current and $B$ is the measurement bandwidth. The shot noise characteristic is similar to that of an undoped single QW with a thin injection barrier [19]. After an excited electron escapes from the SL region, an electron is immediately refilled from the emitter contact. Since the energy of the refilled electron is lower than the blocking barrier, the refilled electron cannot recirculate in the structure. Therefore, each excited electron makes exact one pass though the structure. The optical gain $g$ is thus expected to be unity. We should note that since there is no uncertainty in the refilled process (i.e., the recombination process) [17], the $g=1$ in this structure results 
TABLE I

THE STRUCTURE AND PERFormance PARAMETERS OF THE SLIP AND THE 30-PERIOD QWIP

\begin{tabular}{l|c|c}
\hline Sample & \multicolumn{1}{|l|}{ SLIP } & QWIP \\
\hline Barrier Material & $\mathrm{Al}_{0_{3} \mathrm{Ga}_{07} \mathrm{As}}$ & $\mathrm{Al}_{0_{25} \mathrm{Ga}_{0,5} \mathrm{As}}$ \\
\hline Barrier Width & $30 \AA$ & $500 \AA$ \\
\hline Well width & $60 \AA$ & $50 \AA$ \\
\hline Dopant density & $5 \times 10^{17} \mathrm{~cm}^{-3}$ & $1.2 \times 10^{18} \mathrm{~cm}^{-3}$ \\
\hline Periods & 15 & 30 \\
\hline Peak wavelength $\lambda_{p}$ & 9.3 & 9.1 \\
\hline Cutoff wavelength $\lambda_{c}$ & 10 & 9.8 \\
\hline Spectral bandwidth & $19 \%$ & $20 \%$ \\
$\Delta \lambda$ (FWHM)/ $\lambda_{p}(\%)$ & & \\
\hline Peak responsivity $(\mathrm{mA} / \mathrm{W})$, & $70 \mathrm{~mA} / \mathrm{W}$, & $92 \mathrm{~mA} / \mathrm{W}$, \\
Bias voltage & $0.05 \mathrm{~V}$ & $0.9 \mathrm{~V}$ \\
& $105 \mathrm{~mA} / \mathrm{W}$, & $320 \mathrm{~mA} / \mathrm{W}$ \\
& $0.45 \mathrm{~V}$ & $2 \mathrm{~V}$ \\
\hline Dark current $(\mathrm{A})$, & $1.9 \times 10^{-7} \mathrm{~A}$ & $1.7 \times 10^{-6} \mathrm{~A}$ \\
Bias voltage & $0.05 \mathrm{~V}$ & $0.9 \mathrm{~V}$ \\
\hline Detectivity $\left(\mathrm{cm} \sqrt{H_{z}} / \mathrm{W}\right)$, & $4.2 \times 10^{9}$, & $4.7 \times 10^{9}$ \\
Bias Voltage & $0.05 \mathrm{~V}$ & $0.9 \mathrm{~V}$ \\
\hline
\end{tabular}

in $i_{n}^{2}=2 e g I B$ rather than the usual generation-recombination noise characteristics $i_{n}^{2}=4 \mathrm{eg} I B$ observed in QWIP's. Assuming photoelectrons to transport through the same path as that of thermal excited electrons, we expect the noise performance of the background photocurrent also following the same characteristics.

\section{QUANTUM EFFICIENCY AND DETECTIVITY}

With the measurement of responsivity and noise current, we can determine the quantum efficiency and the detectivity of this device. The net quantum efficiency at the peak wavelength can be calculated from

$$
R_{p}=\frac{e \lambda_{p}}{h c} \eta g
$$

where $R_{p}$ is the peak responsivity, $h$ the plank constant, $c$ the speed of light, and $g$ the photoconductive gain . Taking $g=1$ and $R_{p}=105 \mathrm{~mA} / \mathrm{W}$ at $9.3 \mathrm{um}$, we estimated $\eta=1.4 \%$. The net quantum efficiency is composed of two factors, the optical quantum efficiency $\eta_{a}$ and the escape probability $P$ [6]. That is, $\eta=\eta_{a} P$. In a bound-to-continuum QWIP, the $P$ is usually reached unity at the maximum responsivity. In this 15-period SLIP, the optical quantum efficiency determined from the absorbance of the sample with an eight-pass $45^{\circ}$ facet waveguide configuration is about $1.6 \%$. The escape probability at the maximum responsivity is estimated to be about $88 \%$. For a SLIP without a reflection barrier, the typical escape probability is expected to be about $50 \%$ because half of the photoelectrons are emitted to the emitter contact. If we take the mean free path of the photoelectron into account, the escape probability is expected to decrease with increased thickness of the SL layer. Therefore, although the optical quantum efficiency can be increased with a larger period number, the net quantum ef-

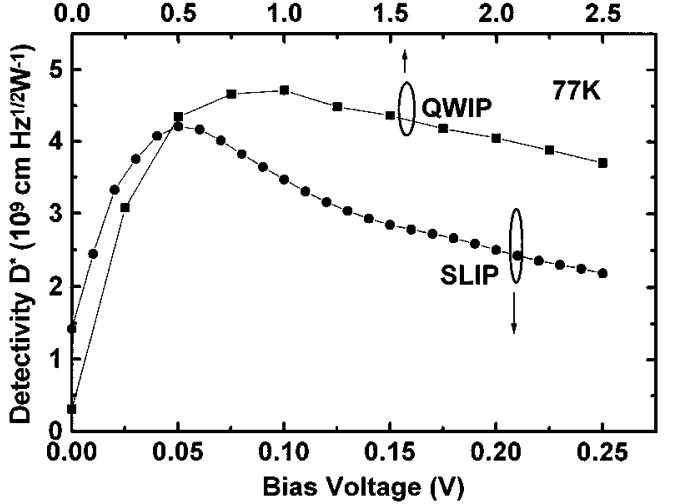

Fig. 8. Detectivity of the SLIP and the 30-period QWIP as a function of bias voltage at $77 \mathrm{~K}$. The decrease of detectivity of both samples is caused by the rapid growth of dark current at high bias voltage.

ficiency may not increase much from the larger period number due to the decrease of the escape probability.

The peak detectivity is defined as

$$
D_{\lambda_{p}}^{*}=\frac{R_{p} \sqrt{A B}}{i_{n}}
$$

where $R_{p}$ is peak responsivity, $A$ the detector area, and $i_{n}$ the noise current. The estimated peak detectivity $D^{*}$ is $3.5 \times 10^{9}$ $\mathrm{cm} \sqrt{\mathrm{Hz}} / \mathrm{W}$ at $V=0.1$ and $T=77 \mathrm{~K}$. At lower temperatures $(<65 \mathrm{~K})$ the detector becomes background limited. The $D_{\mathrm{BLIP}}^{*}$ is estimated about $1.5 \times 10^{10} \mathrm{~cm} \sqrt{\mathrm{Hz}} / \mathrm{W}$.

We compared our results with a 30-period bound-to-continuum QWIP, which has a similar spectral response to our SLIP. The structure and performance parameters of the two devices are summarized in Table I. The voltage-dependence detectivity of the two devices is shown in Fig. 8. We found that the detectivity of the SLIP is comparable to that of the QWIP. However, the SLIP can work at the low bias range $(0-0.3 \mathrm{~V})$ whereas the detectivity of the QWIP is low in this bias range. The optimum bias point of the QWIP is about $0.9 \mathrm{~V}$. The responsivity is $92 \mathrm{~mA} / \mathrm{W}$ and the dark current is $1.67 \times 10^{-6} \mathrm{~A}$ at this bias voltage. On the other hand, the optimum bias voltage for the SLIP is about $50 \mathrm{mV}$. At this bias voltage, the responsivity is $70 \mathrm{~mA} / \mathrm{W}$, and the dark current is $1.9 \times 10^{-7} \mathrm{~A}$. With comparable detectivity and responsivity, the power dissipation of our SLIP is nearly two orders of magnitude smaller than this QWIP.

\section{CONCULUSION}

We have investigated a 15-period superlattice structure for infrared detection with low operating voltage and lower power consumption. A strong photovoltaic response is observed in this asymmetrical structure. We also found a tunable feature of spectral response due to effect of the current blocking barrier in our device. The response spectrum shows voltage dependence around $0 \mathrm{~V}$. At higher bias voltage $(>25 \mathrm{mV})$ the spectra are independent of the bias and are approximately $8-10 \mu \mathrm{m}$ with peak wavelength at $9.3 \mu \mathrm{m}$. Due to the asymmetrical lineshape, the detector still has a responsivity of about $30 \%$ of its peak value at $6 \mu \mathrm{m}$. The measured lineshape of response spectra are similar to the reported absorption spectra of superlattice structures. 
At lower negative bias voltages the response is shifted toward shorter wavelength range. The magnitude of the responsivity also shows a strong dependence on the bias polarity. The responsivity under reverse bias voltages is almost 20 times smaller than that under forward bias voltages. The noise power spectral density of the dark current at $77 \mathrm{~K}$ shows the characteristics of $i_{n}^{2}=2 e I_{d} B$ rather than generation-recombination noise. The peak wavelength detectivity is $D^{*}=3.5 \times 10^{9} \mathrm{~cm} \sqrt{\mathrm{Hz}} / \mathrm{W}$ at $77 \mathrm{~K}$ and $0.1 \mathrm{~V}$ with a responsivity of $85 \mathrm{~mA} / \mathrm{W}$. Background limited performance can be achieved up to $65 \mathrm{~K}$ with $D_{\mathrm{BLIT}}^{*}=1.5 \times 10^{10} \mathrm{~cm} \sqrt{\mathrm{Hz}} / \mathrm{W}$. The lower bias voltage, lower power consumption, and tunable spectral response of the detector should have advantages for practical applications.

\section{REFERENCES}

[1] B. F. Levine, "Quantum-well infrared photodetectors," J. Appl. Phys., vol. 74, pp. R1-R81, 1993.

[2] H. C. Liu, B. F. Levine, and J. Y. Andersson, Eds., Quantum Well Intersubband Transition Physics and Devices. New York: Plenum, 1994.

[3] S. R. Andrews and B. A. Miller, "Experimental and theoretical studies of the performance of quantum-well infrared photodetectors," J. Appl. Phys., vol. 70, pp. 993-1003, 1991.

[4] G. Sarusi, S. D. Gunapala, J. S. Park, and B. F. Levine, "Design and performance of very long-wavelength $\mathrm{GaAs} / \mathrm{Al}_{x} \mathrm{Ga}_{1-x}$ As quantum-well infrared photodetectos," J. Appl. Phys., vol. 76, pp. 6001-6008, 1994.

[5] J. Chu and S. S. Li, "The effect of compressive strain on the performance of $p$-type quantum-well infrared photodetectors," IEEE J. Quantum Electron., vol. 33, pp. 1104-1113, 1997.

[6] B. F. Levine et al., "Photoexcited escape probability, optical gain, and noise in quantum well infrared photodetectors," J. Appl. Phys., vol. 72, pp. 4429-4443, 1992.

[7] L. C. West and S. J. Eglash, "First observation of an extremely large dipole infrared transition within the conduction band of a GaAs quantum well," Appl. Phys. Lett., vol. 46, pp. 1156-1159, 1983.

[8] S. D. Gunapala et al., "Long-wavelength $640 \times 486 \mathrm{GaAs} / \mathrm{AlGaAs}$ quantum well infrared photodetectos snap-shot camera," IEEE. Trans. Electron Devices, vol. 45, pp. 1890-1895, Sept. 1998.

[9] G. Hasnain, B. F. Levine, S. Gunapala, and N. Chand, "Large photoconductive gain in quantum well infrared photodetectors," Appl. Phys. Lett., vol. 57, pp. 608-610, 1990.

[10] E. Pelve et al., "Analysis of the dark current in doped-well multiple quantum well AlGaAs infrared photodetectors," J. Appl. Phys., vol. 66, pp. 5656-5659, 1989.

[11] C. H. Kuan et al., "Hot-electron distribution in multiple quantum well infrared photodetectors," Appl. Phys. Lett, vol. 63, pp. 2091-2093, 1993.

[12] A. Kastalsky, T. Duffield, S. J. Allen, and J. Harbison, "Photovotaic detection of infrared light in a GaAs/AlGaAs superlattice," Appl. Phys. Lett., vol. 52, pp. 1320-1322, 1988.

[13] O. Byungsung et al., "Long-wavelength infrared detection in Kastalsky-type superlattice structure," Appl. Phys. Lett., vol. 57, pp. 503-505, 1990

[14] C. S. Wu et al., "Novel GaAs/AsGaAs multiquantum-well Schottkyjunction device and its photovoltaic LWIR detection," IEEE Trans. Electron Devices, vol. 39, pp. 234-240, 1992.
[15] K. M. S. Bandara et al., "GaAs/AlGaAs superlattice miniband detector with $14.5 \mu \mathrm{m}$ peak response," Appl. Phys. Lett., vol. 60, pp. 3022-3024, 1992.

[16] S. D. Gunapala, B. F. Levine, and N. Chand, "Bound to continuum superlattice miniband long wavelength $\mathrm{GaAs} / \mathrm{Al}_{x} \mathrm{Ga}_{1-x}$ As photodetectors," J. Appl. Phys., vol. 70, pp. 305-308, 1991.

[17] V. I. Dalal, "Simple model for internal photoemission," J. Appl. Phys., vol. 42, pp. 2274-2279, 1971.

[18] M. Helm et al., "Infrared absorption in superlattices: A probe of the miniband dispersion and the structure of the impurity band," Phys. Rev. $B$, vol. 48, pp. 1601-1606, 1993.

[19] K. M. S. V. Bandara, B. F. Levine, and M. T. Asom, "Tunneling emitter undoped quantum-well infrared photodetector," J. Appl. Phys., vol. 74, pp. 346-350, 1993

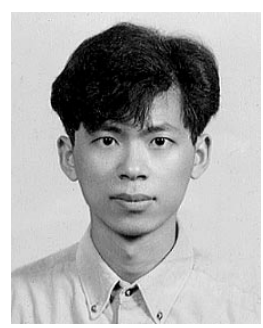

Mao-Chieh Hsu ( $\mathrm{S}^{\prime} 00$ ) was born in Taipei, Taiwan, R.O.C. He received the M.S. degree in physics from National Taiwan University (NTU), Taipei, Taiwan, in 1992 . He is currently pursuing the Ph.D. degree in electrical engineering at NTU.

His current interests are in the development and characterization of infrared devices using the intersubband transitions in quantum well or superlattice structures.

Yao-Fong Hsu received the M.S. degree in electrical engineering from National Taiwan University, Taipei, Taiwan, R.O.C., in 1998.

He is currently serving in Taiwan Army.

Shih-Yan Lin received the M.S. degree in electrical engineering from National Taiwan University (NTU), Taipei, Taiwan, R.O.C., in 1997. He is currently pursuing the Ph.D. degree in electrical engineering at NTU.

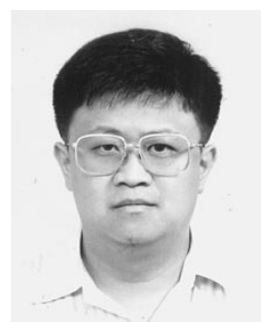

Chieh-Hsiung Kuan (M'95) was born in Taipei, Taiwan, R.O.C., in 1962. He received the B.S. degree in electrical engineering from National Taiwan University (NTU) in 1985, and the M.S.A. and $\mathrm{Ph} . \mathrm{D}$. degrees in electrical engineering from Princeton University, Princeton, NJ, in 1990 and 1994, respectively. His Ph.D. studies focused on the dark current and noise characteristics of the infrared hot-electron transistors and cooperated with the U.S. Army Laboratory, Fort Monmouth, NJ.

$\mathrm{He}$ joined the Department of Electrical Engineering, NTU, in 1994, as an Associate Professor. His current research interests include infrared photodiode for room temperature operation, quantum well infrared photodetector and laser, and problems related to measuring and suppressing the noise in the detectors.

Dr. Kuan is a member of Phi Tau Phi. 